\title{
A holistic model for coastal flooding using system diagrams and the Source-Pathway-Receptor (SPR) concept
}

\author{
S. Narayan ${ }^{1}$, S. Hanson ${ }^{1}$, R. J. Nicholls ${ }^{1}$, D. Clarke ${ }^{1}$, P. Willems ${ }^{2}$, V. Ntegeka ${ }^{2}$, and J. Monbaliu ${ }^{2}$ \\ ${ }^{1}$ Faculty of Engineering and the Environment, University of Southampton, Southampton, England, UK \\ ${ }^{2}$ Department of Civil Engineering - Hydraulics Section, Katholieke Universiteit Leuven, Leuven, Belgium
}

Correspondence to: S. Narayan (sid.narayan@gmail.com)

Received: 15 September 2011 - Revised: 16 March 2012 - Accepted: 18 April 2012 - Published: 14 May 2012

\begin{abstract}
Coastal flooding is a problem of increasing relevance in low-lying coastal regions worldwide. In addition to the anticipated increase in likelihood and magnitude of coastal floods due to climate change, there is rapid growth in coastal assets and infrastructure. Sustainable and integrated coastal flood management over large areas and varying coastline types cannot be simply treated as local combinations of flood defences and floodplains. Rather, a system level analysis of floodplains is required to structure the problem as a first step before applying quantitative models. In this paper such a model is developed using system diagrams and the Source-Pathway-Receptor (SPR) concept, to structure our understanding of large and complex coastal flood systems. A graphical systems model is proposed for the assessment of coastal flood systems with regard to individual elements and their topological relationships. Two examples are discussed - a unidirectional model for a large-scale flood system, and a multi-directional model for a smaller-scale system, both based on the Western Scheldt estuary. The models help to develop a comprehensive understanding of system elements and their relationships and provide a holistic overview of the coastal flood system. The approach shows that a system level analysis of floodplains is more effective than simple topographic maps when conveying complex information. The models are shown to be useful as an apriori approach for making the assumptions about flood mechanisms explicit and for informing inputs to numerical models.
\end{abstract}

\section{Introduction}

Coastal floods from extreme events are without doubt among the costliest natural disasters worldwide (Kron, 2008). Further, coastal zones are becoming more risky as the probabilities and consequences of these flood events increase due to climate change and development pressures. Therefore, analysis of floods in these regions is essential in order to understand risks and minimise losses. Several regions today adopt risk-based approaches to designing coastal protection by analysing the probabilities and consequences of flood events. To understand these probabilities and consequences, coastal managers and decision-makers use a variety of flood maps based on numerical models of flood events (de Moel, 2009; EXCIMAP, 2007).

These models and maps improve our understanding of the hydraulics of flood events and help reduce losses during extreme flood events through efficient flood risk reduction strategies. However, widespread damage still occurs repeatedly despite excellent forecasts and numerical models being available. Events such as Storm Xynthia in France (Kolen, 2010), the July 2007 floods in the UK (Pitt, 2008) and Hurricane Katrina in the US (Seed et al., 2008), though well-forecasted and modelled, caused considerable damage in their respective regions, and revealed shortcomings in our understanding of coastal flood systems. These shortcomings have more to do with the application of numerical and quantitative models than with the models themselves.

While numerical flood models can be applied with great detail and at very fine spatial resolutions, these are often too expensive in terms of data requirements and computational time for use in large and complex coastal flood systems. Also, the nature of these flood systems poses several 
challenges in ensuring that application of these models is based on a comprehensive understanding of the system. This paper aims to address these issues by proposing a systems level analysis of coastal floodplain behaviour.

\section{Challenges in understanding coastal flood systems}

\subsection{Introduction}

A coastal flood system, in the context of this paper, is defined as a geographical region comprising all natural and human related elements potentially affected by a defined flood event. There are several challenges in developing a comprehensive understanding of a complex coastal flood system, including the size of the system, the number of components, including different land uses, and administrative and political boundaries. At present flood risk studies rely almost entirely upon numerical flood models for their analyses of the coastal flood system. However, considerations of financial and computational expense make it difficult to obtain and use accurate data or detailed numerical models at large scales. Added to this are the dangers of missing out key inputs and features that may result in an incomplete definition of the coastal system within numerical models.

\subsection{Size and complexity}

Hurricane Katrina in 2005 in New Orleans, USA is one of the costliest coastal flood disasters in history (Seed et al., 2008). Though bigger than expected and prepared for, the event provided several key lessons for flood risk management. Due to the size and complexity of the New Orleans coastal defence system as well as the relevant organisations, there was a lack of overview on the state of flood defences prior to the event. This led to weaknesses and maintenance gaps in some dyke sections being overlooked that aggravated flooding in the region (Seed et al., 2008). A similar lack of overview on emergency response measures and flood defences led to aggravation of damage during the July 2007 floods in England (Pitt, 2008) and Storm Xynthia in France in 2010 (Kolen, 2010). A methodology formalising knowledge about the flood system, state, importance and relevant organisational structure would have helped reduce the aggravation of flood damage in these instances. This information would also allow better representation of gaps and weak links within numerical models of the flood system.

\subsection{Unexpected pathways and unnatural boundaries}

Such a methodology for the formalisation of flood system knowledge will also help identify the existence of potential, unexpected flood pathways that could aggravate flood damage. This issue was brought to the fore during Storm Xynthia in 2010 where development contrary to spatial planning laws and a lack of knowledge of potential flood routes within the system caused authorities and inhabitants to be taken by surprise (Kolen, 2010). Additionally, flood maps and models are often constrained by administrative delineations that do not recognise the full extent of the natural flood system, especially where the systems cross political boundaries. The existence and location of flood routes in urban regions are of particular importance in numerical models, and such information may be missed in low resolution models. The challenge here is to capture simply, yet effectively, the natural flood system in its entirety with key information on all potential flood routes. A formalised understanding of the full extent of the system will also be invaluable when applying models to regions bounded by administrative and political boundaries rather than natural flood boundaries.

\subsection{Diverse land-use types and inter-dependencies}

Coastal flood systems typically consist of a large number of land-use types and an equal variety of stake-holders and experts. Providing a platform for experts from diverse fields to arrive at a shared understanding of the managed flood system is a difficult task. This is further complicated by interdependencies between the flood system elements. For instance, natural coastal habitats such as mangroves and saltmarshes provide protection during flood events, but these are themselves often affected by flood events; and a change in their state during one storm will affect the flood risk of linked areas during subsequent events. Capturing these interdependencies across the flood system and quantifying the effects on flood risk due to changes in the states of particular elements are significant challenges. While many models take key relationships between system elements into account, the process is often static and becomes difficult for larger systems. A simplified model of the topological relationships will allow users to understand effects on the system as particular elements change, or as new information about these elements is obtained.

\subsection{Summary of challenges}

The size and complexity of coastal flood systems means that there are several challenges associated with gaining a comprehensive understanding of these systems. An inexpensive but rigorous and comprehensive model of the coastal flood system is essential not just for understanding these systems but also for planning and designing flood risk reduction measures. In order to overcome the challenges described, such a model should also be able to integrate important information on different types of elements across the system and provide an overview of the relevant topological relationships and inter-dependencies between these elements. Ultimately, this model should be able to inform subsequent numerical models so as to provide a complete picture of all relevant inputs, elements and features within the system being modelled. 


\section{Current practice in coastal flood risk studies}

\subsection{Conceptual descriptions of coastal flooding}

A popular conceptual model for the description of coastal flooding is the Source-Pathway-Receptor (SPR) concept (Holdgate, 1979). Placed within broader frameworks such as the Driver-Pressure-State-Impact-Response (DPSIR), the SPR allows specific descriptions of the state of coastal flood defence pathways (Evans et al., 2004). The SPR concept has its origins in environmental engineering to describe the flow of environmental pollutants from a source, through different pathways to potential receptors (Holdgate, 1979). It was subsequently adopted for coastal flooding by the UK Environment Agency (H R Wallingford, 2002) to describe the propagation of a flood from a source through flood defences (pathways) to the floodplain beyond (receptors) (Fig. 1).

Coastal flood risk studies generally analyse the physical characteristics of flooding in terms of two components: (a) the hydraulic loading and failure behaviour of structural and non-structural coastal flood defences, and; (b) the hydraulic propagation of flood waters into the landward floodplain (Safecoast, 2008). Coastal flood defences prevent or reduce the entry of coastal flood water into the system. The extent of the floodplain is then dictated by the quantity of water let in by the defence system and local topography.

The pathways of flooding in such an application are limited to coastal defence systems, with every other element in the system being considered a receptor. This does not allow the analysis or description of non-defence system elements or their topological relationships. Therefore, though effective for the analyses of coastal defence systems, this approach does not allow flood risk reduction strategies to address the challenges in large coastal systems (described in Sect. 2).

Recent flood risk studies recognise the coastal floodplain as being a coastal flood system, with inter-linked and interdependent elements. In the UK, the RASP study (Sayers et al., 2002) and the Foresight report on Future Flooding (Evans et al., 2004) recognised the influence of inter-linked elements within the flood system. For instance, these studies consider the role of non-defence elements such as channel vegetation and land-use such as urban habitats in modifying flood event probabilities. The Foresight study used the SPR to describe the role of non-defence elements of the flood system. Subsequent descriptions of the coastal flood system have all been based on a similar concept with minor variations (e.g. Bakewell and Luff, 2008; FLOODSite Consortium, 2009a, b; North Carolina Division of Emergency Management, 2009). Though non-defence elements are considered in these studies, non-local scale system features and relationships relevant to flood management - such as drainage systems or natural habitats potentially acting as flood sinks, were not captured effectively. Further, at a regional to national scale, detailed numerical analysis of the role of individual elements is not practical. It is difficult to capture the topological complexity

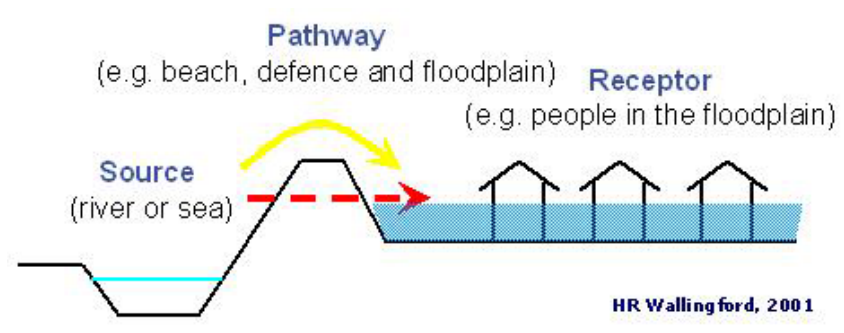

Fig. 1. The Source-Pathway-Receptor (SPR) model in flooding analysis (H R Wallingford, 2002, obtained from www.floodsite.net, (C) H R Wallingford, 2001).

of a flood system and integrate different element and landuse types within flood models. The Foresight report identified key research priorities for coastal flood systems. These include (a) a framework for integrated modelling of different elements within the flood system and (b) the need for a tool that captures the interactions of system elements, especially at the broad scale, to inform decision and policy makers (Evans et al., 2004).

\subsection{Systems models for coastal flooding}

Geographical flood maps, while very useful in conveying specific information to particular users, are not well-suited for describing complex systems and topological relationships between elements. A tool or framework, to address the research priorities described, will need to convey large amounts of complex information to users and experts from diverse fields. System diagrams are a popular and effective means of conveying topological relationships and feedbacks between elements in various fields such as electricity and transport infrastructure. A widely known use of a topological system map is the London Underground map. This map provides a diagram of the functional relationships of the underground railway system, despite not conveying scale, depth and distance travelled. Such topological maps can be very useful in communicating complex information at the right level of abstraction (Kramer, 2007).

The Environment Agency (2009) suggested a systems model to effectively describe and analyse large-scale geomorphological systems consisting of several elements with complex interactions. This form of conceptualisation allows the easy identification of key features and relationships. Importantly, being scale-independent the systems model captures the influence of important elements at non-local scales. The model also helps formalise our understanding of the system and removes the black box nature of existing models (EA, 2009). In the field of coastal flooding, fault tree analyses have been conducted on specific coastal flood defences (e.g. de Boer et al., 2007). However, no such model exists for an entire coastal flood system to date. 


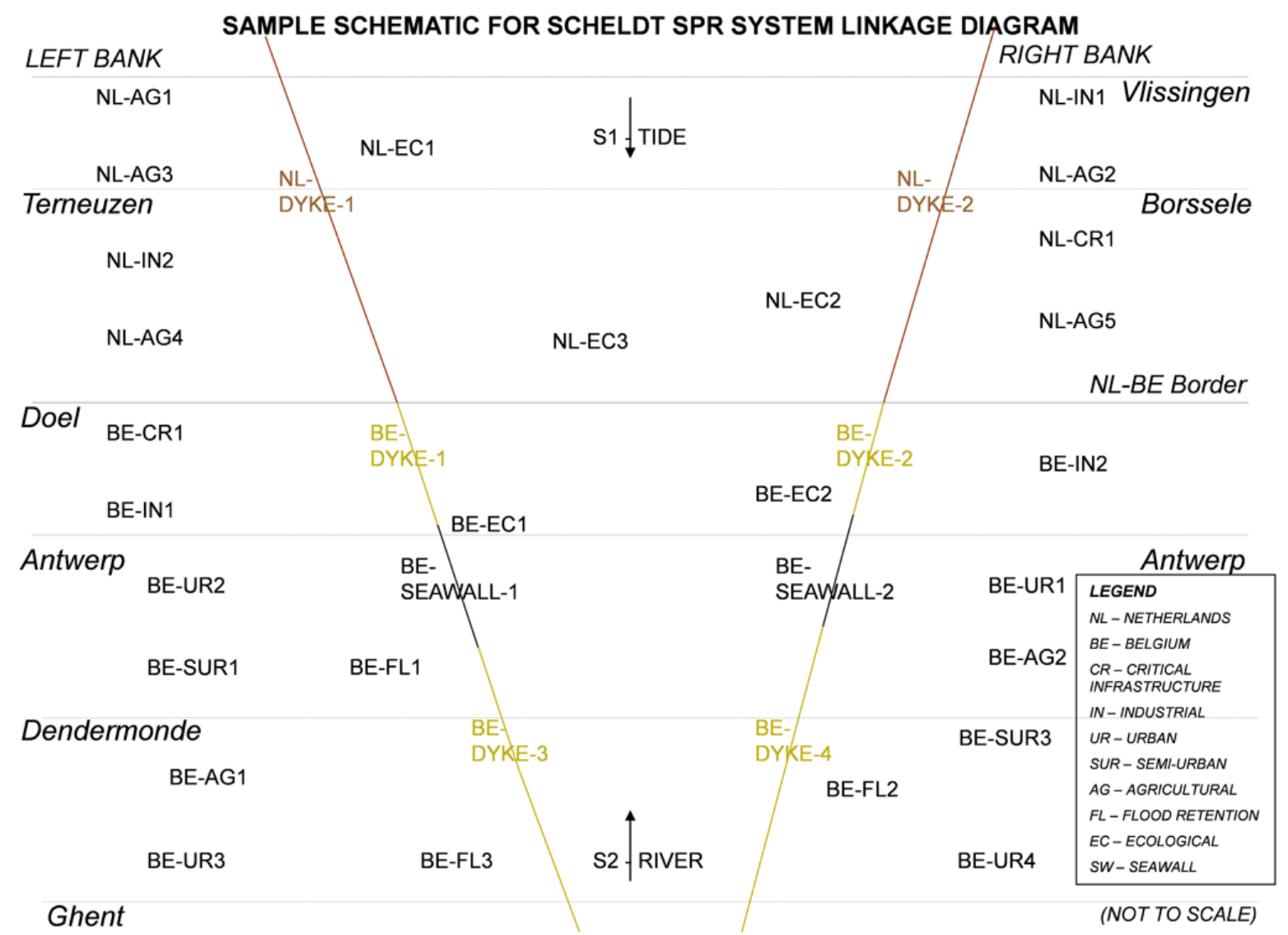

Fig. 2. Simplified stretched schematic of the Western Scheldt showing the considered flood system (100 yr flood $+3 \mathrm{~m}$, planar water level, no defences), the elements within the flood system including assumed defence sections, political boundaries and the (two) sources of flooding (based on data from Google Earth) (based on steps 1 and 2 of Methodology in Sect. 4.2).

\section{The a priori systems model}

\subsection{Introduction}

Here, we describe the building of a systems model for coastal flood systems, based on the principles of the RASP (Sayers et al., 2002), Foresight (Evans et al., 2004) and coastal geomorphology (EA, 2009) studies. The SPR approach is combined with the concept of system diagrams to better recognise key system features and topological relationships between elements. Hence, a more holistic understanding of the flood system is achieved, prior to analysis of these sites with numerical models. The authors intend that this model be used prior to numerical models, in order to inform users of the state, constituent elements and inter-dependencies of the coastal flood system being modelled. It will be applied to sites currently being evaluated under the EU THESEUS project (www.theseusproject.eu). THESEUS is a Europewide project that aims to integrate analyses of the engineering, ecological and socioeconomic aspects of coastal flood system management for better solutions to the problems of climate change and sea level rise.

\subsection{Methodology}

The combination of SPR with system diagrams is a powerful way of collating a comprehensive description of the state of the flood system, its elements and their relationships. The initial focus is on identifying the receptors and building up a network of pathways. A key principle in this approach is the recognition that the definitions of "pathways" and "receptors" are relative, rather than fixed as in earlier applications. Thus, all components of a system may simultaneously function as pathways to "downstream" receptors and as receptors in their own right.

The aim of the systems model is to allow event-specific analyses of the coastal flood system. To ensure that no eventspecific analysis is in danger of missing out potential sources, pathways or receptors, the model is developed iteratively, with the first iteration performed for the most extreme event considered. This ensures that any analysis starts with the largest considered system extent. Subsequent analyses of the system for lesser events will derive their system extents from the largest possible extent obtained in the first iteration. The 


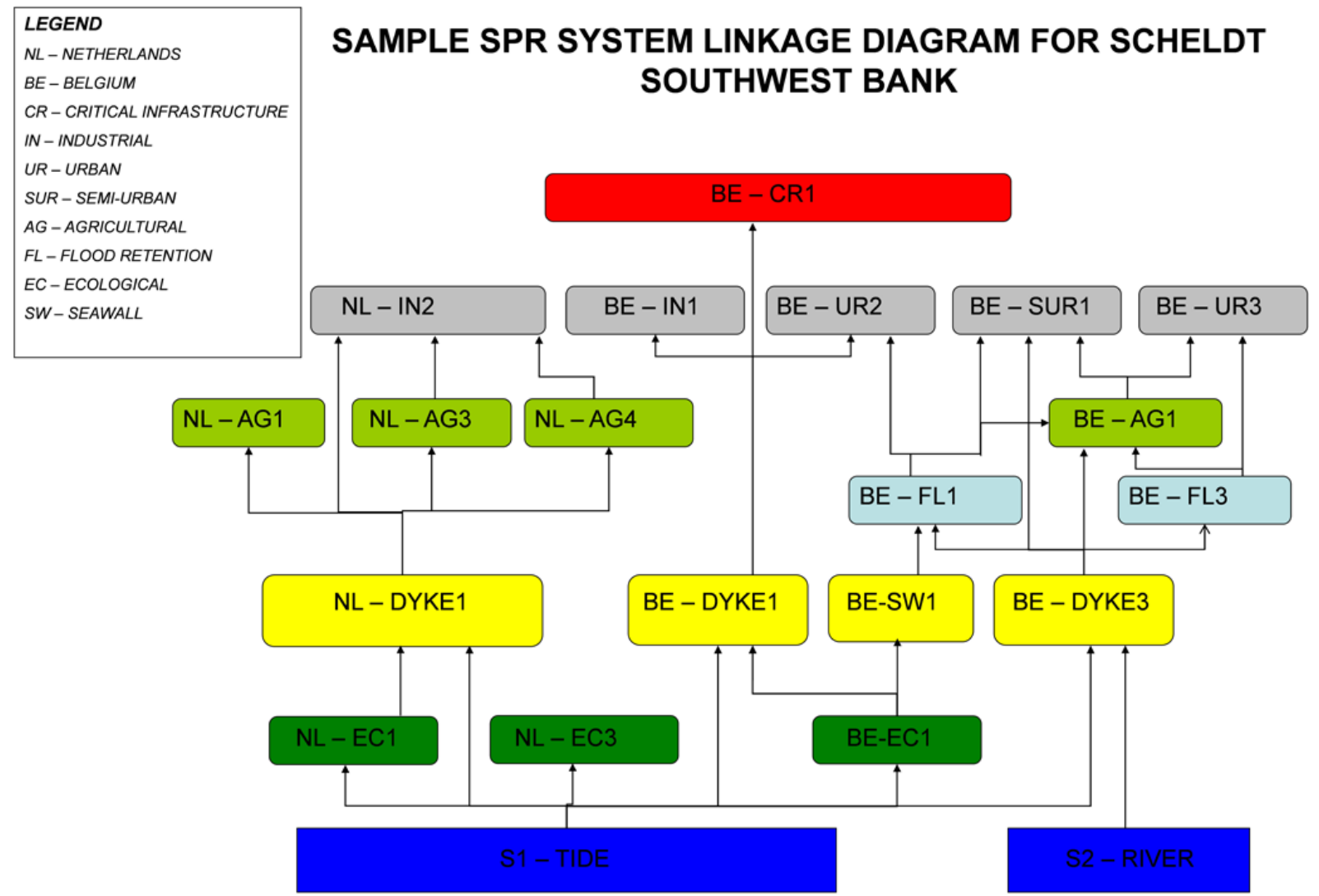

Fig. 3. Systems model for the large-scale flood system (southwest bank of the Western Scheldt), based on the schematic shown in Fig. 2. The sources are indicated as S1 and S2, and the system elements are labelled based on their land-use classification, as shown in Fig. 2 (based on steps 3 and 4 of Methodology in Sect. 4.2).

generic methodology for the first iteration development of the system model is described below.

Step 1: The boundaries of the coastal flood system are first decided using a planar water level model for the most extreme water level being considered. This is done under the assumption of a worst-case scenario where complete failure (or absence) of engineered defences is assumed. This assumption will indicate the full extent of the natural flood system and ensure that all system elements are included in subsequent analyses.

Step 2: Once the natural system extent has been delineated, all elements within the flood system, including flood defences, are mapped as unique entities classified based on land-use. This may be done manually on a map or using a GIS-based software. Individual elements may be of different sizes, since the model is intended to be scale-independent. This allows flexible selection of elements within the system that may be of particular importance to flooding, such as engineered flood defences, flood defences in urban buildings, natural habitat sinks or other such non-local scale features. Since this classification is done after application of the planar water level model, it has no influence on the extent of the system. This classification based on land-use provides a platform for further event-specific analyses of the consequences to specific receptors. Potential effects of changes to land-use within the system during and between flood events may also be analysed. Figures 2 and 4 (discussed in Sects. 4.3 and 4.4, respectively) provide examples of this step.

Step 3: The next step is to define the relationships between the identified elements. At this stage, a link is identified between any two elements if the elements share a geographical boundary. Links between engineered flood defences and the rest of the system are also identified on the same basis. Flood compartments created by these defences can therefore be studied as part of the bigger natural flood system, rather than as isolated sub-systems. The elements and links are then schematised, and a systems map is drawn that maintains as much spatial representation as is practical. The move from a geographical map to a systems map allows easy, quick and comprehensive analyses of the topological relationships between different elements regardless of their location or size. Figures 3 and 5 (discussed in Sects. 4.3 and 4.4., respectively) give examples of this step.

Step 4: Once the complete system diagram is built, the sources of flooding are identified on all boundaries and, if necessary, within the system boundaries. These sources are 


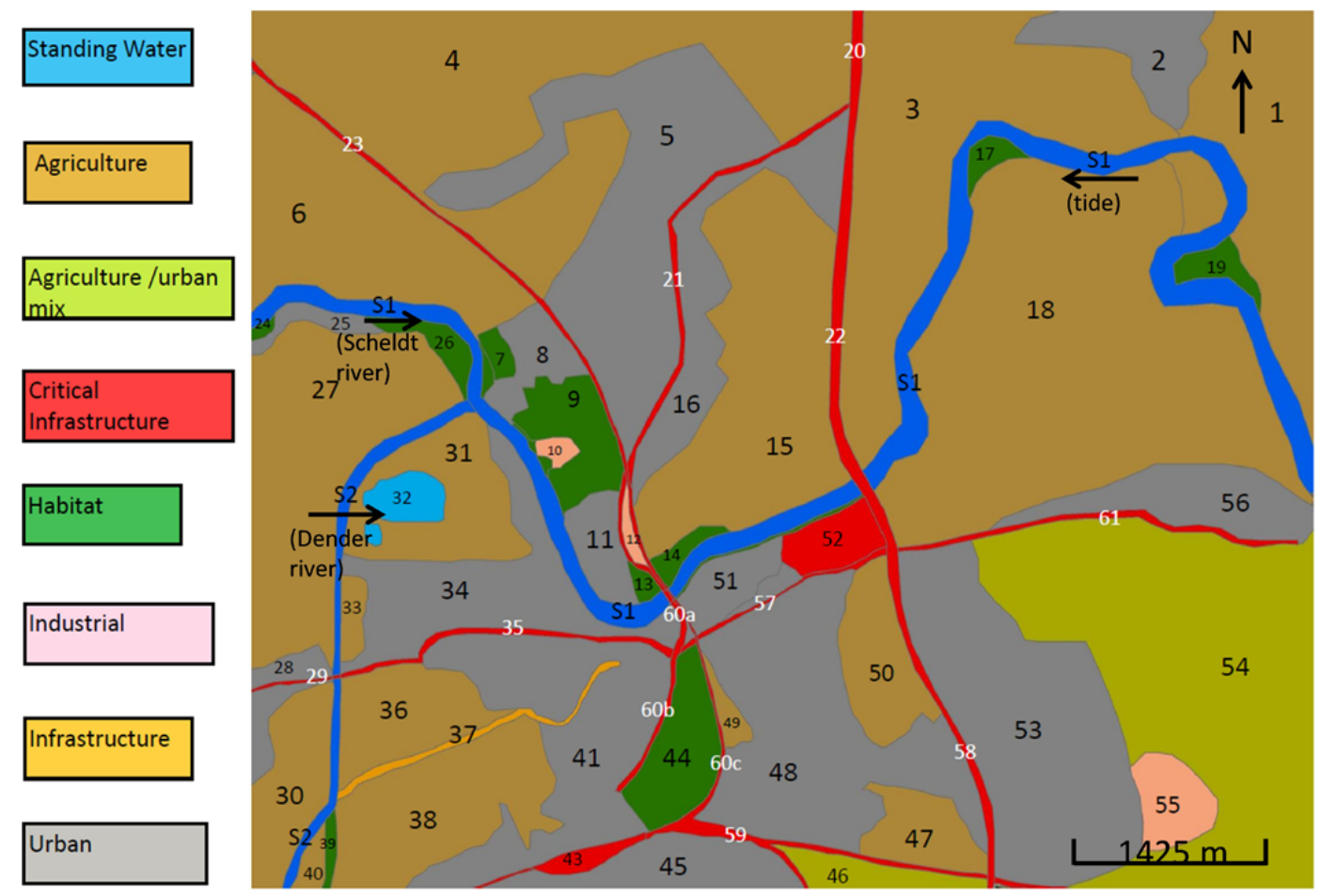

Fig. 4. Dendermonde flood system map for the maximum considered flood extent ( $100 \mathrm{yr}$ flood $+3 \mathrm{~m}$, planar water level, no defences $)$ with numbered elements classified by land-use (Area A - flood system north of S1; Area B - flood system between S1 and S2; Area C - flood system south of S1, east of S2) (based on data from Google Earth). The regions collectively correspond to element "BE-UR3" in Fig. 3 (based on steps 1 and 2 of Methodology in Sect. 4.2).

also schematised and all links between them and directly connected system elements are identified.

In this way a complete systems map of the natural coastal flood system is obtained, with all the elements under consideration identified. Their relationships regarding possible flood routes, all possible sources of flooding and their directions and points of entry are illustrated. A lesser flood event may result in a modification of system extent and element links depending on the relative flood depth for that event. Though not performed in this paper, this modification can be achieved later with flood data, and/or numerical models and form the basis of further flood risk analyses. The ordered progression of systems analysis from the most extreme events to lesser flood events ensures that key receptors and flood pathways are not missed out during flood risk analyses.

To illustrate the approach, two examples of the systems model are presented here: (1) a unidirectional representation of a regional-scale flood system across two countries; and (2) a multi-directional representation of a small-scale flood system within the region of the first model. The system extent in both cases was decided using a planar water level corresponding to the maximum considered water level (a 100-yr flood plus a freeboard of $3 \mathrm{~m}$ to allow for extreme increase in water levels).

\subsection{Large-scale systems model (the Western Scheldt)}

A systems model was built for the Western Scheldt estuary in Europe. The estuary is $350 \mathrm{~km}$ long and flows through Belgium and the Netherlands. The tidal influence reaches the city of Ghent in Belgium. The estuary also experiences river flooding in combination with high tides at upstream locations. Several urban, semi-urban, industrial and agricultural regions are present on either bank in both countries, and these are protected by dikes and seawalls. The estuary and its banks also hold a number of protected natural habitats that are in conflict with human activity (Bouma et al., 2005). Hence, the Scheldt estuary is a complex and interesting flood system and case study.

The systems model for the Scheldt is built using the methodology described in Sect. 4.1. For this study, the flooding on either bank of the Scheldt is considered to be independent. A systems model is built for the entire length of the southwest bank of the estuary. The area is classified into geographical elements based on the predominant land-use. Linear sections of defence elements, differentiated on the basis of element type and design levels, are assumed for the purposes of illustration. Two sources of flooding are considered: high tides and high river runoff.

Figure 2 shows the schematic of the Scheldt used here. This schematic is built based on steps 1 and 2 of the 


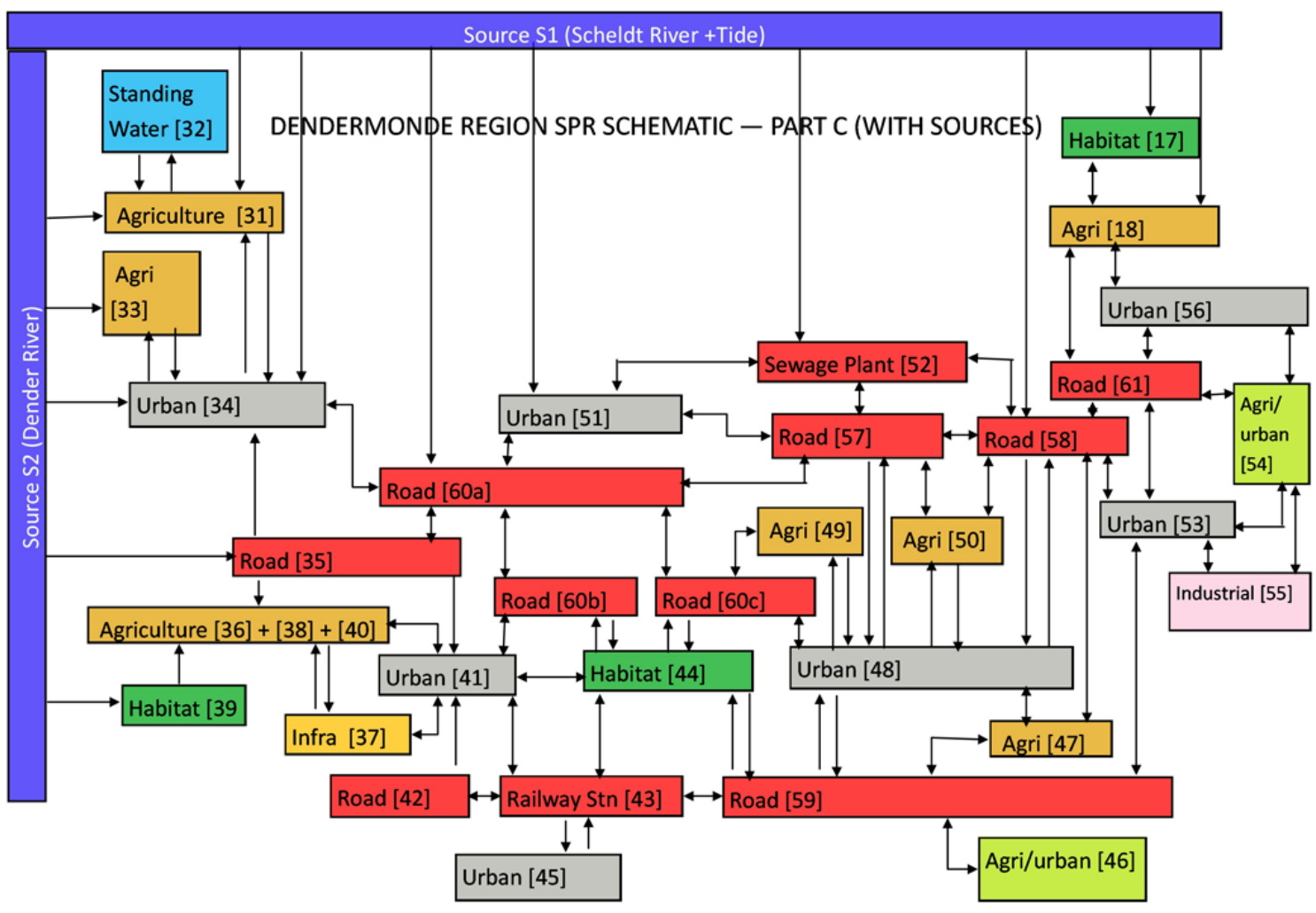

Fig. 5. Systems model for the small-scale flood system (Area $C$ of the Dendermonde flood system shown in Fig. 4). The elements and sources for Area $\mathrm{C}$ are represented as per the flood system map in Fig. 4 (based on steps 3 and 4 of Methodology in Sect. 4.2).

methodology described in Sect. 4.2. Since both sources enter the flood system from the river, an elongated shape is used to approximate the river basin. Figure 3, built using the rules outlined in steps 3 and 4 of the methodology, shows the system SPR diagram for the elements on the right side of the bank of the Scheldt (going upstream). Though the diagram is built independent of administrative boundaries, the elements are given suffixes "NL" or "BE" to indicate their political regions.

The system diagram in Fig. 3 allows a rapid broad-scale assessment of this large-scale flood system. The diagram makes explicit the considered system extent, system elements and element relationships. Relationships between specific defence elements and urban regions, such as between elements "BE Dyke1" and "BE SW1" and the city of Antwerp are easily identified. These relationships will not be as obvious on a flood map, especially when the defence elements and the urban regions are at considerably different scales. The model also captures the relative roles of elements as pathways as well as receptors. In the map in Fig. 3, the habitat region of "BE EC1", defence element "BE Dyke1" and critical infrastructure, "BE CR1" are all potential receptors of flood-induced change in their own right. Additionally, there is a link between natural habitat "BE EC1" and critical infrastructure element "BE CR1" through defence element "BE Dyke1". Thus, the habitat and defence elements become pathways when the receptor is the critical infrastructure. This illustrates the fact that a change in state of the habitat will have an effect on the infrastructure element. Such links between spatially disjoint elements are easily identified with this systems model. In this manner, specific weak links may be pinpointed for more detailed studies. The systems map is therefore useful in prioritising further investigations, while making sure that the entire system is captured and understood at all stages of the analysis process. As another example, Fig. 3 highlights the vulnerability of elements connected to defence element "BE Dyke3" to a combination of tidal and riverine flood sources. These include the urban region of Dendermonde, labelled "BE UR3". The combination of sources at this point indicates this area as one where more in-depth investigation would be beneficial. Hence, this area is the subject of the second, smaller-scale systems model.

The large-scale systems model may be used by coastal authorities and managers in both the countries to arrive at a common understanding of the shared flood system, by understanding the relationships between elements on either side of the border. Coastal authorities or concerned stake-holders for specific receptors can use the map as a rapid assessment of the topological links between their receptor of interest and other system elements. This will facilitate integrated policy and decision-making regarding flood zoning and protection measures. Such a map of large-scale systems can also be used 
to identify critical and important pathways within the system, and provide a rational framework for prioritising further research needs.

\subsection{Small-scale systems model (of Dendermonde)}

The multi-directional SPR system model permits greater detail and spatial representation. The procedure for building the model is the same as in the previous case. Once the system extents are decided for the worst-case scenario, the Dendermonde flood system is divided into three broad compartments where flooding is assumed to occur independently. This is done based on the nature and location of the sources and the local geomorphology. As in the first example, elements in each compartment are categorised by their predominant land-use. Due to the finer scale and multi-directional nature of the sources, a greater degree of spatial accuracy is maintained during the schematisation.

Figure 4 shows a map with the flood system divided into three compartments based on the geomorphology and classified based on land-use. This figure is built using the rules described in steps 1 and 2 of the methodology described in Sect. 4.2. Figure 5, based on steps 3 and 4 of the described methodology, shows the systems diagram for Area $\mathrm{C}$ of the flood system, situated south of the main estuary.

The systems diagram in Fig. 5 allows a quick and comprehensive a priori analysis of the Dendermonde flood system. Since the defences are assumed to fail, the full natural extent of the flood system is made clear. The model makes clear the possibility, however small, that the railway station, "[43]" may be flooded not just from the north or the west, but from other directions as well by flood routes through other system elements. It thus provides a comprehensive platform for analysing the range of flood routes within complex systems. All system elements are presented on the same systems map, making it easy to identify relationships between habitat elements and surrounding urban elements. For instance, habitat element "[44]", if designed as a flood retention region, could mitigate flood risk to the station and surrounding road links. Similarly, agricultural and standing water elements "[32]" and "[31]" may be used to mitigate flood risk to urban element "[34]". Such systems analyses can help to focus the efficiency of the subsequent application of numerical models for flood risk studies. The SPR network potentially draws out detailed, element-specific questions of interest concerning the given flood system that encourage a better model design and application.

\subsection{Discussion}

In both case studies, the systems model is effective in providing complex information that will be difficult to convey on a simple flood map. It serves to inform users of the assumptions and considerations being made in subsequent numerical models. It is observed from the two case studies that progression to a multi-directional model at smaller scales is necessary to obtain a complete representation of the flood system - similar to the increase in feature representation in numerical models with increasing resolution. This is mainly due to the finer resolution of the sources and elements, necessitating the representation of multi-directional sources and element links. However, since this systems model is scaleindependent, it is possible to aggregate different elements or ignore certain links for a more simplified model. Thus, the user can choose only to represent the key elements and links of interest for the given analysis. The main advantage of doing this in the systems model, rather than in numerical models, is that the assumptions made in the process of aggregating elements or ignoring links becomes explicit and can easily be corrected or modified if necessary. It is therefore useful as an a priori model in the flood modelling process.

\section{Conclusions}

Despite the availability of excellent forecasting and numerical flood models, there remain gaps in our understanding of the coastal flood system, and hence in our applications of these models. However, the size, complexity and diversity of these systems pose considerable challenges in gaining a comprehensive understanding of flood system elements and their relationships. The detail that such studies will require makes it impractical to rely solely on numerical models. In this paper, an a priori systems model for coastal flood systems is developed based on the concepts of SPR and system diagrams. The model is capable of providing complex information about the system and element relationships in a robust and effective manner. It is also a powerful means of making key explicit assumptions and considerations about the system, providing users a comprehensive understanding of their flood system. The systems model is not meant to replace flood maps or fully quantitative numerical models; instead, it is intended to be used alongside these, to ensure that a comprehensive understanding of the flood system before quantitative modelling.

The formalisation of the model building process with a generic rule-based algorithm is being done at present. When finished, this will allow the model to be applied to any type of coastal system at any scale or level of detail. In this paper the model is applied to coastal flood systems at two scales. It is expected to be most useful in large and complex coastal systems where detailed numerical models are expensive and data for calibration and validation scarce. The model provides a structured and integrated overview of both flood systems, avoiding compartmentalisation of system elements into artificial sub-units. Since all the elements are mapped onto the same platform, along with the relevant relationships, the model is very useful in developing a common understanding of the systems amongst experts from different fields. Importantly, this understanding can be achieved prior to numerical 
modelling. This allows the formulation of element-specific questions about flood risks, impacts and management strategies. Appropriate numerical models may then be applied to explore the resulting questions.

The systems model, by developing the key topological relationships between elements, also provides an excellent foundation for analysing changes in flood risk across the system, due to changes in particular elements. Further work on this aspect of the model will attempt to quantify the uncertainties associated with different weak links within a system. These results, validated by results from numerical flood models, will be used to map the sensitivity of the system to different elements and pathways. Finally, the model offers great potential for identification of critical components and analyses of system failure pathways, all of which will be explored in later stages of this study.

Acknowledgements. The authors are very grateful to the two anonymous reviewers for their patience and constructive comments in the process of preparing this paper. The support of the European Commission through the project "Innovative Technologies for safer European coasts in a changing climate" (THESEUS), Contract 244104, FP7.2009-1, www.theseusproject.eu, is gratefully acknowledged.

Edited by: F. J. Mendez

Reviewed by: two anonymous referees

\section{References}

Bakewell, I. and Luff, S.: North London strategic flood risk assessment - Final Report, DEFRA, UK, 2008.

Bouma, T. J., Vries, M. B. D., Low, E., Kusters, L., Herman, P. M. J., Tánczos, I. C., Temmerman, S., Hesselink, A., Meire, P., and Regenmortel, S. V.: Flow hydrodynamics on a mudflat and in salt marsh vegetation: Identifying general relationships for habitat characterisations, Hydrobiologia, 540, 259-274, 2005.

de Boer, E., Kortenhaus, A., and van Gelder, P.: Comparison of coastal flooding probability models for flood defences in proceedings of the 5 th coastal structures international conference, CST07, Venice, Italy, July 2007, 639-644, 2007.

de Moel, H., van Alphen, J., and Aerts, J. C. J. H.: Flood maps in Europe - methods, availability and use, Nat. Hazards Earth Syst. Sci., 9, 289-301, doi:10.5194/nhess-9-289-2009, 2009.

Environment Agency: Characterisation and prediction of largescale, long term change of coastal geomorphological behaviours: Final science report, Environment Agency, Bristol, UK, 2009.
Evans, E., Ashley, R., Hall, J., Penning-Rowsell, E., Sayers, P., Thorne, C., and Watkinson, A.: Foresight Future Flooding: Scientific Summary: Volume I - Future risks and their drivers, Office of Science and Technology, London, 2004.

EXCIMAP: Handbook on good practices for flood mapping in Europe, European Exchange Circle on Flood Mapping, 2007.

FLOODSite Consortium: Methodology for a dss to support longterm flood risk management planning, H R Wallingford, 144 pp., 2009a.

FLOODSite Consortium: available at: http://www.floodsite. net/html/work_programme_detail.asp?taskID=8, last access: June 2009b.

Gouldby, B., Sayers, P., Mulet-Marti, J., Hassan, M., and Benwell, D.: A methodology for regional-scale flood risk assessment, P. I. Civil Eng.-Wat. M., 161, 169-182, 2008.

H R Wallingford: Risk, performance and uncertainty in flood and coastal defence - a review, SR 587, 2nd Edn., H R Wallingford, Wallingford, UK, 2002.

Holdgate, M. W.: A perspective of environmental pollution, Cambridge University Press Cambridge, UK, 1979.

Kolen, B., Slomp, R., and Balen, W. V.: Learning from French experiences with storm Xynthia, Rijksoverheid.nl, 2010.

Kramer, J.: Is abstraction the key to computing, Communications of the ACM, Vol. 50, April 2007, New York, USA, 2007.

Kron, W.: Coasts-the riskiest places on earth, Proceedings of the International Conference on Coastal Engineering (ICCE), 3-21, 2008.

North Carolina Division of Emergency Management: North Carolina sea level rise risk management study - potential impacts, risk assessments and management strategies, 2009.

Pitt, M.: Learning lessons from the 2007 floods: An independent review by Sir Michael Pitt, London: The Pitt Review, Cabinet Office, 2008.

Safecoast: Coastal flood risk and trends for the future in the north sea region, synthesis report, Safecoast project team, The Hague, 136 pp., 2008.

Sayers, P., Hall, J., Rosu, C., Chatterton, J., and Deakin, R.: Risk assessment of flood and coastal defences for strategic planning (RASP) - a high level methodology, Environment Agency, UK, 2002.

Scottish Government: Strategic environmental assessment (sea) of draft plan for offshore wind energy in Scottish territorial waters, Volume 1: Environmental report, 2010.

Seed, R., Bea, R., Abdelmalak, R., Athanasopoulos-Zekkos, A., Boutwell, G., Briaud, J. L., Cheung, C., Cobos-Roa, D., Ehrensing, L., and Govindasamy, A.: New Orleans and Hurricane Katrina. I: Introduction, overview, and the east flank, J. Geotech. Geoenviron., 134, 701-717, 2008. 